\title{
X-ray imaging of atomic nuclei
}

\author{
Jie Xu, Jia He, Yi Ding and Jun Luo*
}

\begin{abstract}
Optically imaging atomic nuclei is a long-sought goal for scientific and applied research, but it has never been realized so far. We integrate aberration-corrected scanning transmission electron microscopy (STEM), the bremsstrahlung generation of $\mathrm{X}$-ray photons, and the energy-dispersive $\mathrm{X}$-ray spectroscopic (EDS) receiving and mapping of the photons into a new microscopy method of optical imaging, by which atomic nuclei of different materials are successfully imaged with X-ray photons. Moreover, this imaging method is shown to be workable with different STEM instruments and be capable of distinguishing atomic nuclei of different elements and resolving imaged size differences of atomic nuclei with the order of magnitude as small as $\mathbf{1} \mathbf{~ p m}$. Therefore, it is a general method that can image atomic nuclei and their evolutions in materials science, chemistry and physics.
\end{abstract}

Keywords: atomic nuclei, X-ray imaging, bremsstrahlung, aberration-corrected scanning transmission electron microscopy

\section{INTRODUCTION}

Super-resolution optical imaging methods, such as scanning near-field optical microscopy (SNOM), stimulated emission depletion (STED) microscopy and stochastic optical reconstruction microscopy (STORM), have been playing important roles in materials science, chemistry, physics and biology [1-6]. For instance, the spatial resolutions of SNOM, STED and STORM reach 1, 20 and $20 \mathrm{~nm}$, respectively, leading to the optical reveal of nanodimension with great details [3-6]. However, to date, no methods of optical imaging can observe atomic nuclei.

Atomic nuclei not only are the cores of substances but also directly participate in bremsstrahlung (namely deceleration radiation), nuclear magnetic resonance (NMR), nuclear scattering, and spontaneous and induced nuclear reactions including nuclear fission, fusion, spallation and decay, all of which are significant for scientific research and practical applications, such as nuclear power, nuclear medicine and NMR imaging [7,8]. Therefore, extensive efforts have been devoted to studying atomic nuclei for over 100 years $[7,8]$. However, using photons to image atomic nuclei has never been realized so far. This difficulty is due to that it is highly challenging to achieve simultaneously the emission of photons from atomic nuclei or their related effects, the reception of the photons, the distinguishment of the photons from those emitted by the electrons around the atomic nuclei, and the precise positioning of the atomic nuclei [3-6].

Fortunately, aberration-corrected scanning transmission electron microscopy (STEM) can provide an opportunity. Its electron beam is capable of positioning sample sites with the pm-scale precision, and the interactions of the electron beam with the atomic nucleus and the electrons of an atom can emit bremsstrahlung X-ray photons and characteristic ones, respectively [9-17]. Moreover, the bremsstrahlung and the characteristic Xray photons can be received and distinguished by the detectors of energy-dispersive X-ray spectroscopy (EDS) in aberration-corrected STEM [9-17]. The above functions can be performed simultaneously by modern instruments of aberration-corrected STEM [9-14]. However, up to now, no studies have been reported on integrating these functions together to realize the X-ray imaging of atomic nuclei (ANXRI).

Herein, we propose and realize a new microscopy type of optical imaging for ANXRI by integrating aberrationcorrected STEM, the bremsstrahlung generation of X-ray photons, and the EDS receiving and mapping of the photons. By this ANXRI method, atomic nuclei of different materials are successfully imaged. Moreover, this imaging method works with different STEM instruments and has the abilities to distinguish atomic nuclei of different elements and resolve imaged size differences of atomic nuclei at the pm scale. This work offers opportunities for imaging atomic nuclei and their evolutions in materials science, chemistry and physics.

Center for Electron Microscopy and Tianjin Key Lab of Advanced Functional Porous Materials, Institute for New Energy Materials \& Low-Carbon Technologies, School of Materials Science and Engineering, Tianjin University of Technology, Tianjin 300384, China

* Corresponding author (email: jluo@email.tjut.edu.cn) 


\section{EXPERIMENTAL SECTION}

\section{Materials}

The samples of Pd, Pt-coated nanoporous gold (NPG), denoted as NPG-Pd-Pt, and NPG without Pd or Pt were synthesized by dealloying, copper under-potential deposition and galvanic replacement reactions, the details of which have been previously reported $[9,11]$. The $\mathrm{SrTiO}_{3}$ sample is standard $\mathrm{SrTiO}_{3}$ purchased from Hefei Ke Jing Materials Technology Co., Ltd, China, and its thinning was done by mechanical polishing and then argon ion milling in a Gatan instrument of Precision Ion Polishing System 691.

\section{Characterizations}

The STEM images and the EDS signals for ANXRI were obtained using the aberration-corrected STEM modes of JEOL JEM-ARM $300 \mathrm{~F}$ at $300 \mathrm{kV}$ and FEI Titan Cubed Themis G2 300 at $200 \mathrm{kV}$. JEOL JEM-ARM 300F contains two EDS detectors, each of which has an active area of $100 \mathrm{~mm}^{2}$. FEI Titan Cubed Themis G2 300 contains four EDS detectors, each of which has an active area of $30 \mathrm{~mm}^{2}$. The corresponding EDS mapping was performed with their standard softwares. In the EDS detection, the STEM instrument of JEOL JEM-ARM 300F itself gives the characteristic EDS peaks of Ti and Fe, FEI Titan Cubed Themis G2 itself gives the ones of Fe and Co, and the grids to hold samples give the ones of $\mathrm{Cu}$. The electron energy-loss spectroscopy (EELS) measurement of the $\mathrm{SrTiO}_{3}$ thickness was performed using an EELS spectrometer of Gatan Enfinium ER 977 installed in FEI Titan Cubed Themis G2 300, the well-established EELS theory and the Gatan DigitalMicrograph (DM) software $[9,18]$.

\section{Calculations}

All calculations were performed with density functional theory (DFT) in the DMol3 code. The Perdew-BurkeErnzenhof functional was used to treat the generalized gradient approximation. A double numerical plus polarization (DNP) was used as the basis set. The spin-unrestricted method was used for all calculations. The convergence tolerance of energy is $2 \times 10^{-5} \mathrm{Ha}$, and the maximum force and displacement are $0.004 \mathrm{Ha}^{-1}$ and $0.005 \AA$, respectively. The real-space global orbital cutoff radius was set to be $5.0 \AA$. The interval of the grid used to calculate the Coulombic potential distributions was set to be $0.1 \AA$, which is the maximum value of the interval available in the software and corresponds to the highest grid resolution. This limited grid resolution caused the slight asymmetry of the Coulombic potential distribution of each atomic nucleus. For the cell optimization, the Brillouin zone was sampled by $10 \times 10 \times 10 \mathrm{k}$-points.

\section{RESULTS AND DISCUSSION}

Based on our previous studies with STEM and EDS [912], our idea about the ANXRI method was originally inspired by the working mechanism of atomic force microscopy (AFM) [19]. As displayed in Fig. 1a, b, when AFM is working, its tip scans the surface of a sample and is displaced by the interaction between the tip and the surface. The tip displacement at each scanned position is recorded, and mapping the displacements at all scanned positions gives a topographic image or even an atomicresolution image of the sample surface [19].

An incident electron in the electron beam of STEM is like an AFM tip. Similar to Fig. 1a and b, Fig 1c and d show that when the electron beam scans an atomic nucleus and its vicinity, the interaction between some incident electrons and the nucleus decelerates the electrons, causing bremsstrahlung and thus the emission of corresponding X-ray photons [13]. The photons represent the energies lost by the incident electrons and can be received and recorded by EDS detectors, which produce continuous count backgrounds for EDS spectra [13]. The EDS-recorded X-ray photon counts are like the recorded displacements of an AFM tip. Thus, if we map the photon counts at all scanned positions around the nucleus center, an image of the nucleus can be obtained. Moreover, similar to adjusting the force between the tip and the sample surface in AFM, when bremsstrahlung X-ray photons with higher (or lower) energies are employed for mapping, an atomic nucleus image with smaller (or larger) diameters can be attained, as shown by the boxed images in Fig. 1c and d. This is because higher-energy photons are emitted when the electron beam is closer to the nucleus center and thus lose higher energies. It should be noted that the main idea with the Coulombic field and its energy distribution in Fig $1 c$ and $d$ is based on the quantum mechanics. It will be confirmed later by calculations of DFT, which is recognized to be a computational quantum mechanical modelling method.

Experimentally, we used aberration-corrected STEM instruments to perform the ANXRI idea. Their electron beams are superfine probes with diameters smaller than individual atoms, such as $0.82 \AA$ of JEOL JEM-ARM 300F and $0.6 \AA$ of FEI Titan Cubed Themis G2 300, and their EDS spectra consist of characteristic count peaks and continuous count backgrounds [9-14,20-28]. The peaks mainly count the amounts of characteristic X-ray photons emitted by the electrons of sample atoms, while the 
a

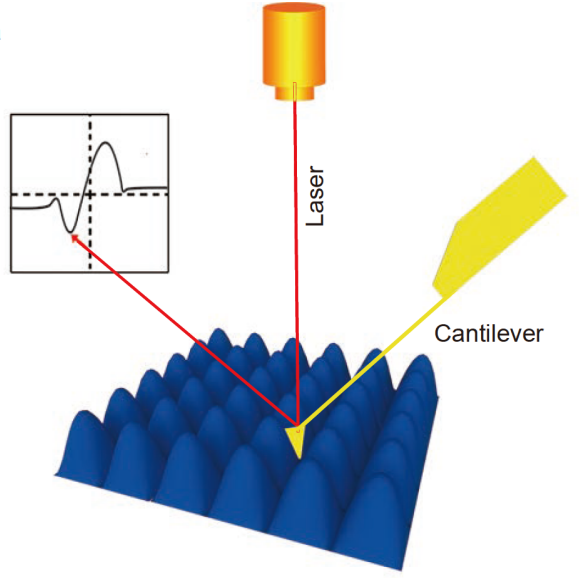

c

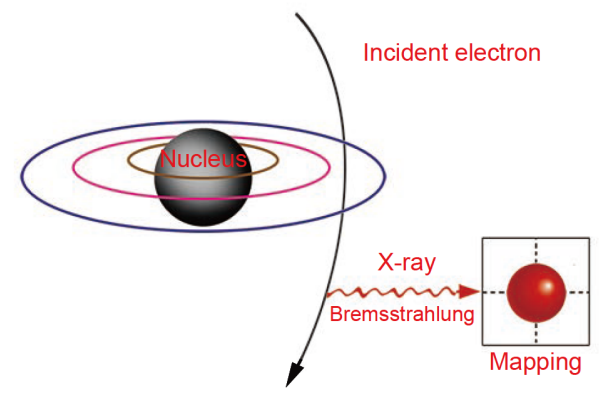

b

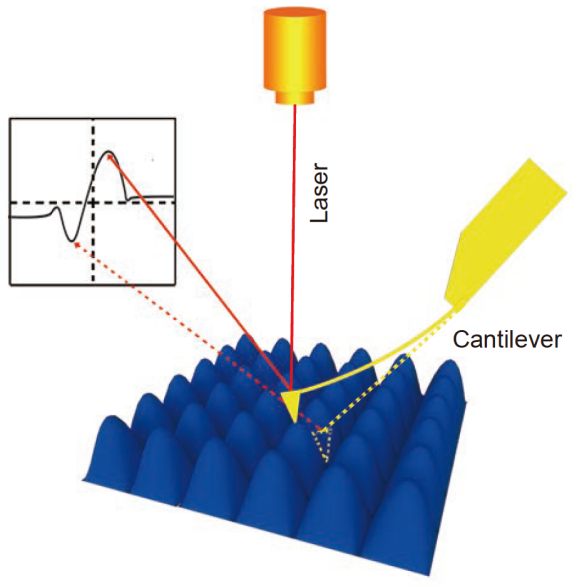

d

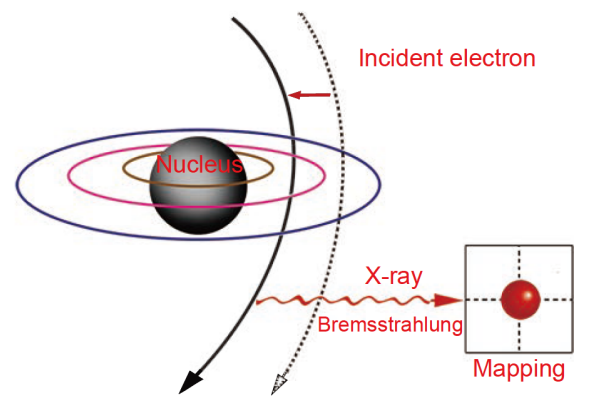

Figure 1 Working mechanisms of AFM and ANXRI. (a, b) Schematic images for the mechanism of AFM when the tip of its cantilever scans a valley and a peak, respectively, on the surface of a sample. During the scanning, the tip is displaced, and its displacements are recorded with the laser beam, giving the topographic curves in the boxes. (c, d) Schematic images for the proposed mechanism of ANXRI when two incident electrons of its electron beam pass by two positions close to and closer to, respectively, the center of an atomic nucleus. The circles represent the Coulombic-field energy distribution of the nucleus. Due to the Coulombic field, the two incident electrons are decelerated, producing bremsstrahlung and thus emitting X-ray photons [13].

backgrounds count the amounts of only bremsstrahlung X-ray photons emitted by the interaction between the incident electrons and atomic nuclei of samples [9-14,2028]. It should be noted the bremsstrahlung radiation can also occur between the incident electrons and the electrons of sample atoms. When the incident electrons have energies $\leq 300 \mathrm{keV}$, the electron-electron bremsstrahlung is safely negligible compared with the electron-nuclei one $[16,17]$. Thus, it is valid that our work does not consider the electron-electron bremsstrahlung, because the incident electrons in our work have the energies of 200 or $300 \mathrm{keV}$. Fig. 2a shows an elemental mapping image of NPG-Pd-Pt [9]. This image clearly gives the elemental distribution in each atom column by characteristic peak counts of $\mathrm{Au}, \mathrm{Pd}$ and Pt. In contrast, the counts in the $E_{1}$ and $E_{2}$ ranges in Fig. $2 \mathrm{~b}$ are those of bremsstrahlung Xray photons. Thus, we used them to perform mapping for the ANXRI idea. The results are shown in Fig. $2 \mathrm{c}$ and $\mathrm{d}$.

The images in Fig. $2 \mathrm{c}$ and $\mathrm{d}$ have been filtered by the Wiener method; this filtering has been widely used to remove noise in images without artifacts induced $[9,11,24,25,28]$ (see raw images and details in Figs S1, S2 and Table S1). Many clear dots with high signal-to-noise ratios exist in Fig. $2 \mathrm{c}$ and d. Comparing them with those of Fig. 2a indicates that their positions are the same as those in Fig. 2a. Further, the fast Fourier transform (FFT) patterns of Fig. $2 c$ and $d$ are given in Fig. $2 e$ and $f$, and each of them contains six diffraction spots, which have the same distribution as those of the FFT patterns of Fig. 2a (see details in Fig. S1). These results manifest that the dots in Fig. $2 \mathrm{c}$ and $\mathrm{d}$ have the same periodic structures as that of the atom columns in Fig. 2a. As is well known, any atom column has the same position as its corresponding atomic nuclei. Moreover, Fig. $2 \mathrm{c}$ and $\mathrm{d}$ were made by mapping the counts of the bremsstrahlung X-ray photons produced by the interaction between atomic nuclei and incident electrons. Therefore, the ANXRI idea is realized, and Fig. $2 \mathrm{c}$ and $\mathrm{d}$ show the images of the atomic nuclei in the atom columns of NPG-Pd-Pt.

Because the ANXRI images are actually EDS mapping 

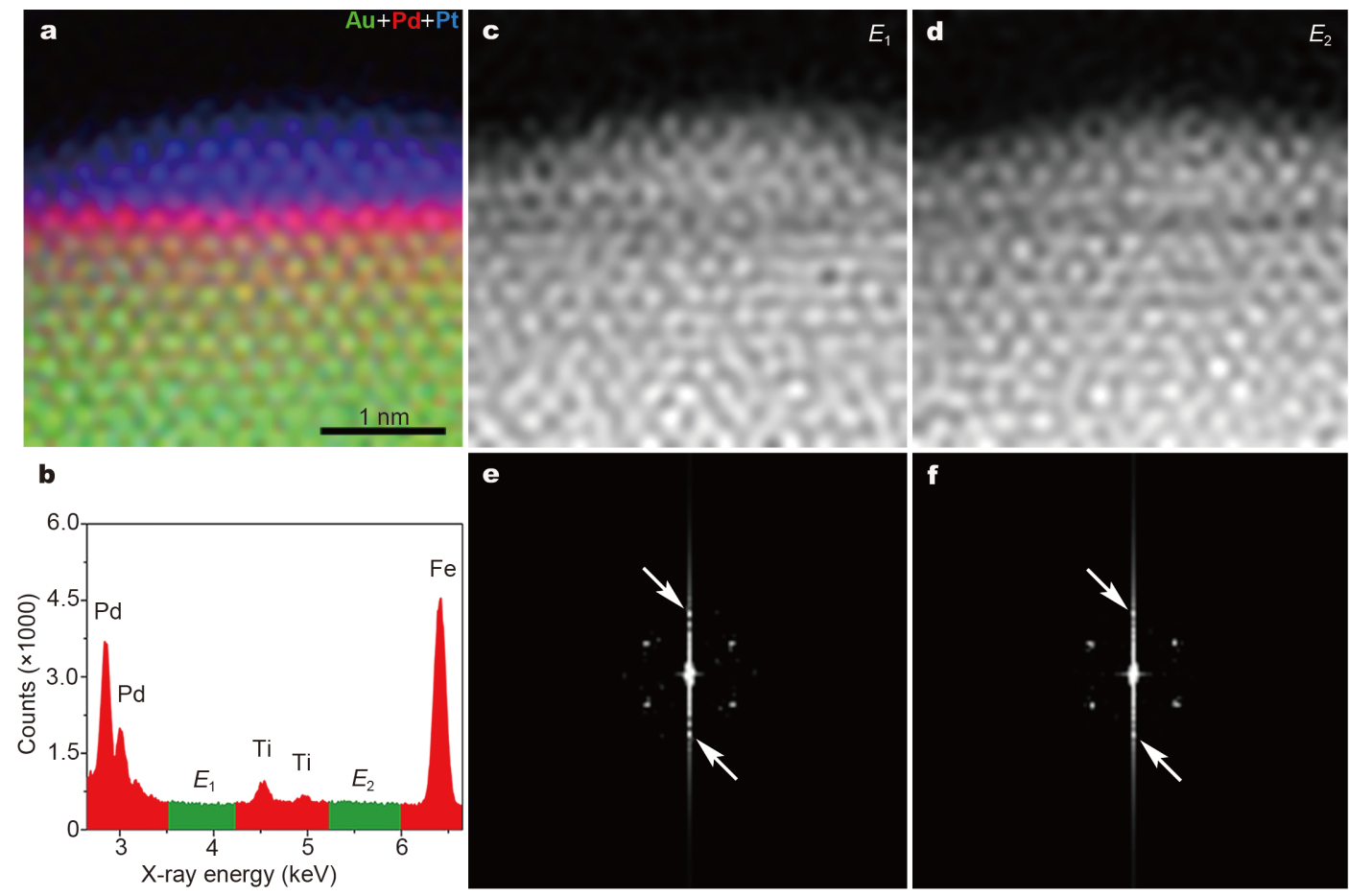

Figure 2 Characterization and ANXRI results of NPG-Pd-Pt, which is a real-world catalyst [9], by JEOL JEM-ARM 300F. (a) Atomically resolved elemental mapping image of the sample using the characteristic EDS peak counts of Au (green), Pd (red) and Pt (blue). Reproduced with permission from Ref. [9]. Copyright 2017, Springer Nature. (b) EDS spectrum from the region in (a). The green-colored energy ranges denoted by $E_{1}$ and $E_{2}$ in the background are from 3.53 to $4.29 \mathrm{keV}$ and from 5.24 to $6 \mathrm{keV}$, respectively, and they have the same width of $0.76 \mathrm{keV}$ (see the Experimental Section and the caption of Fig. S1 for the origins of the characteristic EDS peaks of Ti and Fe and the criteria to choose the energy windows). (c, d) ANXRI images of atomic nuclei obtained by using the counts in the $E_{1}$ and $E_{2}$ ranges in (b) for mapping. The images have the same scale bars as that in (a). (e, f) FFT patterns of (c) and (d), in each of which six diffraction spots exist and two of them are indicated by arrows as examples.

images performed with the bremsstrahlung X-ray photon counts, the physical meaning of their intensity/contrast is the count of the bremsstrahlung X-ray photons emitted at each scanned position on the sample. On the other hand, it should be noted that different energy windows have different energy ranges. The X-ray photons corresponding to a specific higher-energy window have higher energies than those to a lower-energy window. The energies of the bremsstrahlung X-ray photons are from those lost by the incident electrons decelerated by the Coulombic fields of atomic nuclei. When incident electrons are closer to (or farther from) atomic nuclei, they are decelerated by higher (or lower) energies of the Coulombic fields and thus lose higher (or lower) energies to emit higher-energy (or lower-energy) photons. These emission positions are recorded by the scanning of the electron probe and used for constructing the mapping images. Therefore, the emission positions (namely mapping positions) of X-ray photons in ANXRI images qualitatively reflect the energy distributions of the Coulombic fields of atomic nuclei, and the full widths at half maximum (FWHMs) of the intensity profiles of atomic nucleus images are actually a measure on the distributions of the mapping positions.

Of the dots with high signal-to-noise ratios in Fig. 2c and $\mathrm{d}$, we randomly selected 20 dots to measure their peak intensities (PIs) and the FWHMs of their intensity profiles, which represent the individual imaged sizes of the dots (see the measurement details in Figs S2, S3 and Tables S1-S3). The positions of the 20 dots are depicted in Fig. $3 a$ and b. Their FWHM values are shown in Fig. 3c and $\mathrm{d}$, indicating that the FWHM values of the dots corresponding to $E_{1}$ are always larger than those to $E_{2}$. Moreover, these two sets of FWHM values show that their differences have the average of $0.075 \AA$ and the standard deviation of $0.043 \AA$ (see details in Table S3). That is, the difference value between the individual imaged sizes of the atomic nuclei in Fig. $3 a$ and $b$ is $0.075 \pm$ $0.043 \AA$ A namely $7.5 \pm 4.3 \mathrm{pm}$. These results are consistent with the ANXRI prediction shown by Fig. 1c and d, which is that mapping bremsstrahlung X-ray photons with higher (or lower) energies produces an atomic nucleus image with smaller (or larger) diameter. 

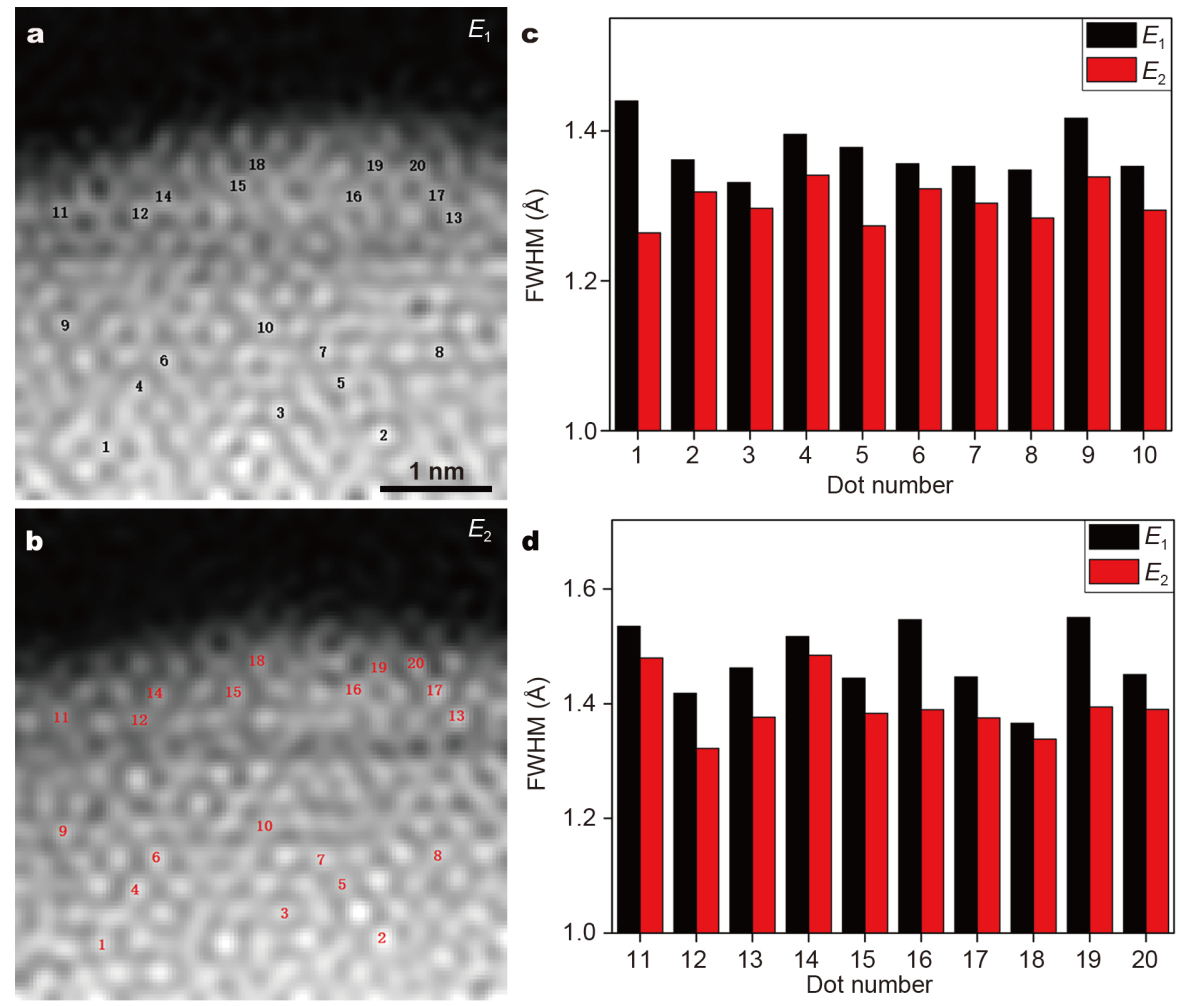

Figure 3 Adjustment of the individual imaged sizes of the atomic nuclei of NPG-Pd-Pt. (a, b) Images of the atomic nuclei, which are identical to Fig. 2 c, d, respectively. In the images, 20 dots are randomly selected and then numbered. (c, d) FWHM values of the 20 dots. The black and the red values were measured in (a) and (b), respectively. It should be noted that all the results in Figs 2 and 3 were achieved using JEOL JEM-ARM 300F on NPG-Pd-Pt, which is a real-world catalyst with nonuniform thickness [9]. We re-performed the experiments with FEI Titan Cubed Themis G2 300 on the well-defined sample of $\mathrm{SrTiO}_{3}$ with uniform thickness and another type of real-world catalyst (NPG without Pd or Pt $[9,29]$ ). Their results also give the images of atomic nuclei and are also consistent with the ANXRI prediction (Figs S4-S8 and Tables S4, S5).

It should be noted that the sample thickness of NPG$\mathrm{Pd}-\mathrm{Pt}$ is not uniform [9]. In contrast, the thickness of the $\mathrm{SrTiO}_{3}$ sample is uniform (Fig. S9 and Table S6). Its elemental mapping image is displayed in Fig. 4a, where the positions of $\mathrm{Sr}$ and $\mathrm{Ti} / \mathrm{O}$ atom columns are shown clearly. With the $E_{1}$ range in Fig. $4 \mathrm{c}$, the atomic nucleus image in Fig. $4 \mathrm{~b}$ was obtained. In Fig. 4b, 128 dots were selected to measure the FWHM values of their intensities (Fig. 4d). Among the values, the ones corresponding to the $\mathrm{Sr}$ columns are all larger than those of $\mathrm{Ti} / \mathrm{O}$ (see more details in Table S7). That is, the individual imaged sizes of the atomic nuclei in the Sr columns are all larger than those in the $\mathrm{Ti} / \mathrm{O}$. The atomic nucleus image made by the $E_{2}$ range in Fig. $4 c$ gives the same result (Fig. S10c, d and Table S8). These results are rational, because an $\mathrm{Sr}$ atomic nucleus has more protons and neutrons than a Ti and an $\mathrm{O}$ together, and the atomic nuclei of different elements have different energy distributions of the Coulombic fields, which will be shown later by DFT. As mentioned before, the distributions of the mapping positions of $\mathrm{X}$ - ray photons in ANXRI images reflect the energy distributions and thus are elemental sensitive. The FWHMs are actually a measure on the distributions of the mapping positions and thus are also elemental sensitive. Therefore, ANXRI is capable of distinguishing atomic nuclei of different elements. This function is very useful, because in most of nuclear scattering and reactions, not the electrons in a material but only its atomic nuclei change and evolve, which cannot be imaged and monitored by characteristic EDS peaks.

Moreover, from the ANXRI results in Figs 3, 4b, d, and Figs S5, S8 and S10, their corresponding imaged size differences are measured to be $7.5 \pm 4.3$ pm (Fig. 3 and Table S3), $16 \pm 5 \mathrm{pm}$ (Fig. $4 \mathrm{~b}, \mathrm{~d}$ and Table S7), $4.7 \pm$ $1.4 \mathrm{pm}$ (Fig. S5 and Table S4), $10 \pm 4 \mathrm{pm}$ (Fig. S8 and Table S5), and $16 \pm 5 \mathrm{pm}$ (Fig. S10c, $d$ and Table S8). This ability indicates that ANXRI is capable of resolving size differences with the order of magnitude as small as $1 \mathrm{pm}$.

To check the above main idea and experimental findings, we performed DFT calculations on $\mathrm{Au}$ and $\mathrm{SrTiO}_{3}$ 

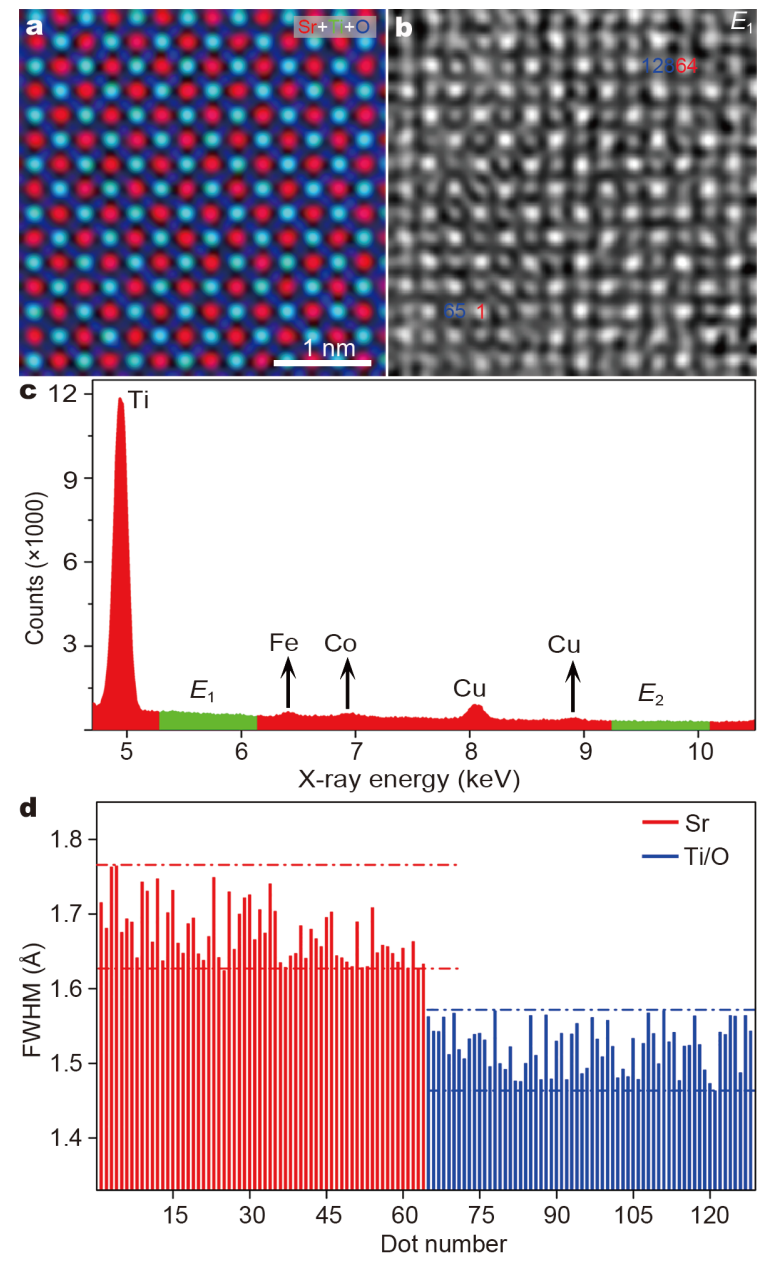

Figure 4 Characterization and ANXRI analysis of the $\mathrm{SrTiO}_{3}$ sample by FEI Titan Cubed Themis G2 300. (a) Atomically resolved elemental mapping image of the sample using the characteristic EDS peak counts of $\mathrm{Sr}$ (red), Ti (green) and $\mathrm{O}$ (blue). Each Ti atom column contains $\mathrm{O}$ atoms with the $\mathrm{Ti} / \mathrm{O}$ ratio of $1: 1$, and thus it is denoted as $\mathrm{Ti} / \mathrm{O}$, for which the overlay of green and blue gives cyan. (b) ANXRI image of atomic nuclei obtained by using the counts in the $E_{1}$ range in (c) for mapping, which has the same scale bar as (a). The dots labeled by the red and the blue numbers are located at the positions of $\mathrm{Sr}$ and $\mathrm{Ti} / \mathrm{O}$ columns, respectively, and the numbers of only four dots are drawn here for clear visualization (128 dots are numbered; please see all 128 numbers in Figs S8 and S10a). (c) EDS spectrum from the region in (a). The $E_{1}$ and $E_{2}$ ranges have the same width of $0.9 \mathrm{eV}$ (see the Experimental Section for the origins of the characteristic EDS peaks of $\mathrm{Fe}, \mathrm{Co}$ and $\mathrm{Cu}$ ). (d) FWHM values of the labeled 128 dots, which all correspond to $E_{1}$. The dashed lines indicate the positions of the maxima and the minima.

(Figs S11 and S12). The results indicate that when an incident electron is close enough to the center of an $\mathrm{Au}$ atomic nucleus, the Coulombic energy affecting the electron increases dramatically, during which a $0.05 \AA$ reduction of distance corresponds to a Coulombic energy increase of several $\mathrm{keV}$. This finding supports the ex- perimental ANXRI result that the individual imaged sizes of $\mathrm{Au}$ atomic nuclei obtained by the two bremsstrahlung energy ranges of 4.85-5.35 keV and 5.55- $6.05 \mathrm{keV}$ have the difference of $0.047 \pm 0.014 \AA$ (Figs S4, S5 and Table S4). The calculation results of $\mathrm{SrTiO}_{3}$ are similar to the above. They also show that the Coulombic energy distributions around the $\mathrm{Sr}$, the $\mathrm{Ti}$ and the $\mathrm{O}$ atomic nuclei are different from each other, suggesting that it is rational to distinguish them by using ANXRI images.

It should be noted that in any imaging method, its core idea makes use of an interaction between an imaging probe and an imaged object. For instance, AFM uses the tip displacement caused by the interatomic force between a tip and a sample surface to image the surface (Fig. 1a, b). High-angle annular dark-field (HAADF) imaging of STEM is another example. It images atomic nuclei in materials by collecting the incident electrons scattered by the Coulombic fields of the atomic nuclei, and this scattering is an interaction between the incident electrons and the atomic nuclei. HAADF is also an imaging method of atomic nuclei. Its way to realize the imaging is to collect the scattered incident electrons, and thus it is not an optical imaging method. Different from HAADF, the ANXRI method images atomic nuclei by collecting X-ray photons generated from the bremsstrahlung interaction between the incident electrons and the atomic nuclei. Therefore, ANXRI is an optical imaging method of atomic nuclei using the bremsstrahlung interaction, which is new and had never been reported before our work. Moreover, because ANXRI is performed with STEM, it can be a bridge between STEM and optical microscopy.

Both of ANXRI and HAADF use the interactions between incident electrons and atomic nuclei, which are caused by the Coulombic fields of the atomic nuclei. Thus, the sizes of atomic nuclei images given by ANXRI and HAADF are both limited by the distributions of the Coulombic fields and have similar orders of magnitudes at around $1 \AA$ (Figs 2c, d, 4b, and Figs S4a and S6a). In addition, when an atomic nucleus is in a material, its Coulombic field is inevitably affected by the electron clouds of its own and adjacent atoms'. Nevertheless, this is the true situation for atomic nuclei in materials. Thus, the atomic nucleus images given by ANXRI and HAADF reflect the true situation of atomic nuclei in materials.

An electron probe in aberration-correct STEM is very fine but still has a size of $0.4-1 \AA$ with tail. As a result, when an electron probe passes by a position close to an atomic nucleus, the distances from the incident electrons in the probe to the nucleus are different from each other, 
and the energies lost by the incident electrons are also different from each other. Thus, the emitted bremsstrahlung X-ray photons have different energies. Nevertheless, the EDS detectors are capable of distinguishing the bremsstrahlung X-ray photons according to their energies, and thus the ANXRI method can choose bremsstrahlung X-ray photons with specific energies to perform the imaging, as shown by Figs $2-4$. Therefore, the influence of the probe size and tail can be negligible in ANXRI.

Generally, different detector geometries can affect the collection efficiency of EDS detectors on bremsstrahlung $\mathrm{X}$-ray photons [13]. Our work used two EDS detector systems with different geometries, one in JEOL JEMARM 300F and the other in FEI Titan Cubed Themis G2 300. The active areas of all the detectors relative to their geometries have been optimized. Thus, the ANXRI images taken with the JEOL and the FEI systems are all atomic-resolution (Figs 2c, d, 4b, and Figs S4j, $\mathrm{m}$ and S7d).

In EDS spectra, general bremsstrahlung backgrounds are smooth continuum, such as the $E_{1}$ and the $E_{2}$ ranges. In contrast, zone axis coherent bremsstrahlung (ZACB) signals are small Gaussian-shaped peaks, similar to small characteristic EDS peaks [13,15]. The aberration-corrected STEM operation with a convergent electron beam effectively reduces the intensities of small ZACB peaks $[13,15]$. Thus, we did not employ ZACB peaks to perform ANXRI.

The probe delocalization effects cause the interaction between the electron probe and the probed atom column to spread to a wider range and thus disturb the atomicresolution imaging and analysis. They can be reduced greatly by the spherical aberration correction and the probe channeling effect, especially when the probed sample is oriented along a low-index zone axis $[13,14]$. These conditions were also used in our work. Therefore, the probe channeling effect and the spherical aberration correction contribute much to the atomic resolution of the ANXRI images.

After their generation from a sample, bremsstrahlung $\mathrm{X}$-ray photons may encounter the other parts of the sample or some components of the STEM instrument, and then some of the photons are absorbed, exciting the sample parts or the STEM components to produce fluorescence [13]. These effects reduce the amounts of the bremsstrahlung X-ray photons reaching the EDS detectors and thus degrade the signal-to-noise ratios of ANXRI images. Further optimization of the STEM instruments may suppress the effects.
Further, we measured the PI values in the ANXRI images of $\mathrm{SrTiO}_{3}, \mathrm{NPG}-\mathrm{Pd}-\mathrm{Pt}$ and NPG, and the results are presented in Table S9 and Figs S13 and S14. Table S9 indicates that the average and standard deviation of the Sr PI values with $E_{1}$ are 193 and 10 , respectively. That is, $\mathrm{PI}_{\mathrm{Sr}, E_{1}}=193 \pm 10$. Besides, $\mathrm{PI}_{\mathrm{Ti} / O, E_{1}}=140 \pm 10, \mathrm{PI}_{\mathrm{Sr}_{2} E_{2}}=174$ \pm 11 , and $\mathrm{PI}_{\mathrm{Ti} / O, E_{2}}=131 \pm 14$. Therefore, $\mathrm{PI}_{\mathrm{Sr}, E_{1}} /$ $\mathrm{PI}_{\mathrm{Ti} / \mathrm{O}, E_{1}}=1.38 \pm 0.12$, and $\mathrm{PI}_{\mathrm{Sr}, E_{2}} / \mathrm{PI}_{\mathrm{Ti} / \mathrm{O}, E_{2}}=1.33 \pm 0.16$. The two ratios equal each other within the error bars. That is, within the current errors of measurement, the change of the selected bremsstrahlung energies does not alter the relative intensity among different atomic columns. Further improvement of the EDS detectors would reduce the errors. Besides, it is obvious that $\mathrm{PI}_{\mathrm{Sr}, E_{1}}>$ $\mathrm{PI}_{\mathrm{Ti} / \mathrm{O}, E_{1}}$, and $\mathrm{PI}_{\mathrm{Sr}, E_{2}}>\mathrm{PI}_{\mathrm{Ti} / O, E_{2}}$. This conforms to the reported bremsstrahlung model [13], which indicates that the number of bremsstrahlung X-ray photons is proportional to the average atomic number. Thus, the ANXRI intensity can also be used to distinguish different elements. In addition, Figs S13 and S14 indicate that in the ANXRI images of NPG-Pd-Pt and NPG, the changes of the intensities and FWHM values against the thicknesses are ruleless. Complex mechanisms may exist in the thickness influence.

\section{CONCLUSIONS}

In this study, ANXRI is for the first time proposed and realized by integrating aberration-corrected STEM, the bremsstrahlung generation of X-ray photons, and the EDS receiving and mapping of the photons. By ANXRI, atomic nuclei in atom columns of NPG-Pd-Pt, NPG and $\mathrm{SrTiO}_{3}$ including heavy metal, light metal and nonmetal elements have successfully been imaged using different STEM instruments. Moreover, the individual imaged sizes of the atomic nuclei are shown to be adjustable by altering the X-ray energies. Further, ANXRI is capable of distinguishing the atomic nuclei of different elements and resolving the imaged size differences of atomic nuclei with the order of magnitude as small as $1 \mathrm{pm}$. These results have been confirmed by DFT calculations. Therefore, ANXRI is a general microscopy method of optical imaging with the pm-scale precision to image and distinguish atomic nuclei. This work provides new opportunities for imaging and studying atomic nuclei and their evolutions in materials science, chemistry and physics, such as nuclear fission, fusion, spallation and decay.

Received 7 February 2020; accepted 30 March 2020;

published online 30 April 2020

1 Gambarotto D, Zwettler FU, Le Guennec M, et al. Imaging cellular 
ultrastructures using expansion microscopy (U-ExM). Nat Methods, 2019, 16: 71-74

2 Barwick B, Flannigan DJ, Zewail AH. Photon-induced near-field electron microscopy. Nature, 2009, 462: 902-906

3 Betzig E, Trautman JK. Near-field optics: microscopy, spectroscopy, and surface modification beyond the diffraction limit. Science, 1992, 257: 189-195

4 Liu Y, Lu Y, Yang X, et al. Amplified stimulated emission in upconversion nanoparticles for super-resolution nanoscopy. Nature, 2017, 543: 229-233

5 Huang B, Wang W, Bates M, et al. Three-dimensional superresolution imaging by stochastic optical reconstruction microscopy. Science, 2008, 319: 810-813

6 Kim S, Yu N, Ma X, et al. High external-efficiency nanofocusing for lens-free near-field optical nanoscopy. Nat Photonics, 2019, 13: 636-643

7 Loveland WD, Morrissey DJ, Seaborg GT. Modern Nuclear Chemistry, Second Edition. New Jersey: JohnWiley \& Sons, Inc., 2015

8 Stock R. Encyclopedia of Nuclear Physics and its Applications. New York: Wiley-VCH Verlag GmbH \& Co. KGaA., 2015

9 Li J, Yin HM, Li XB, et al. Surface evolution of a Pt-Pd-Au electrocatalyst for stable oxygen reduction. Nat Energy, 2017, 2: 17111

10 Zhang L, Han L, Liu H, et al. Potential-cycling synthesis of single platinum atoms for efficient hydrogen evolution in neutral media. Angew Chem Int Ed, 2017, 56: 13694-13698

11 Ma W, Liu X, Li C, et al. Rechargeable $\mathrm{Al}-\mathrm{CO}_{2}$ batteries for reversible utilization of $\mathrm{CO}_{2}$. Adv Mater, 2018, 30: 1801152

12 Liu X, He J, Zhao S, et al. Self-powered $\mathrm{H}_{2}$ production with bifunctional hydrazine as sole consumable. Nat Commun, 2018, 9: 4365

13 Williams DB, Carter CB. Transmission Electron Microscopy-A Textbook for Materials Science. Berlin: Springer, 2009

14 Pennycook SJ, Nellist PD (Ed.). Scanning Transmission Electron Microscopy-Imaging and Analysis. Berlin: Springer, 2011

15 Reese GM, Spence JCH, Yamamoto N. Coherent bremsstrahlung from kilovolt electrons in zone axis orientations. Philos Mag A, 1984, 49: 697-716

16 Kontar EP, Emslie AG, Massone AM, et al. Electron-electron bremsstrahlung emission and the inference of electron flux spectra in solar flares. Astrophys J, 2007, 670: 857-861

17 Haug E. Bremsstrahlung and pair production in the field of free electrons. Z Naturforsch, 1975, 30a: 1099-1113

18 Egerton RF. Electron Energy-Loss Spectroscopy in The Electron Microscope, Third Edition. Berlin: Springer, 2009

19 Haugstad G. Atomic Force Microscopy: Understanding Basic Modes and Advanced Applications. New Jersey: JohnWiley \& Sons, Inc., 2015

20 Urban KW. Studying atomic structures by aberration-corrected transmission electron microscopy. Science, 2008, 321: 506-510

21 Zhou J, Lin J, Huang X, et al. A library of atomically thin metal chalcogenides. Nature, 2018, 556: 355-359

22 Lin J, Zuluaga $\mathrm{S}$, Yu P, et al. Novel $\mathrm{Pd}_{2} \mathrm{Se}_{3}$ two-dimensional phase driven by interlayer fusion in layered $\mathrm{PdSe}_{2}$. Phys Rev Lett, 2017, 119: 016101

23 Yu P, Lin J, Sun L, et al. Metal-semiconductor phase-transition in $\mathrm{WSe}_{2(1-x)} \mathrm{Te}_{2 x}$ monolayer. Adv Mater, 2017, 29: 1603991

24 Ma $\mathrm{C}$, Cheng $\mathrm{Y}$, Chen $\mathrm{K}$, et al. Mesoscopic framework enables facile ionic transport in solid electrolytes for Li batteries. Adv Energy Mater, 2016, 6: 1600053

25 Ma C, Rangasamy E, Liang C, et al. Excellent stability of a lithiumion-conducting solid electrolyte upon reversible $\mathrm{Li}^{+} / \mathrm{H}^{+}$exchange in aqueous solutions. Angew Chem Int Ed, 2015, 54: 129-133

26 Jeong JS, Odlyzko ML, Xu P, et al. Probing core-electron orbitals by scanning transmission electron microscopy and measuring the delocalization of core-level excitations. Phys Rev B, 2016, 93: 165140

27 Zhao Y, Yan X, Yang KR, et al. End-on bound iridium dinuclear heterogeneous catalysts on $\mathrm{WO}_{3}$ for solar water oxidation. ACS Cent Sci, 2018, 4: 1166-1172

28 Sun L, Yan X, Zheng J, et al. Layer-dependent chemically induced phase transition of two-dimensional $\mathrm{MoS}_{2}$. Nano Lett, 2018, 18: 3435-3440

29 Liu P, Guan P, Hirata A, et al. Visualizing under-coordinated surface atoms on 3D nanoporous gold catalysts. Adv Mater, 2016, 28: $1753-1759$

Acknowledgements We thank Prof. Peng Gao from Peking University for providing the $\mathrm{SrTiO}_{3}$ sample, Ketao Zang and Kai Wang from Tianjin University of Technology for assistance in experiments, and Dr. Qiang Xu from Delft University of Technology, Dr. Shu Miao from JEOL, Dr. Guang Yang from FEI and Prof. Li-Min Liu from Beihang University for helpful discussion. The authors also acknowledge the National Supercomputing Center in Shenzhen for providing the computational resources and materials studio (version 7.0, DMol3). This work was financially supported by the National Key R\&D Program of China (2017YFA0700104), the National Science Fund for Distinguished Young Scholars (51825102), the National Natural Science Foundation of China (51971157, 51671145 and 51761165012), and Tianjin Science Fund for Distinguished Young Scholars (19JCJQJC61800).

Author contributions Luo J proposed and designed ANXRI and its schemes of experiments and calculations, and he also supervised the project. Luo J and Xu J co-performed the main experiments and coanalyzed all experimental results, to which Ding Y contributed. He J performed the DFT calculations and analyzed the results, to which Luo J, Ding Y and Xu J contributed.

Conflict of interest The authors declare no conflict of interest.

Supplementary information Detailed analyses and supporting data are available in the online version of the paper. 


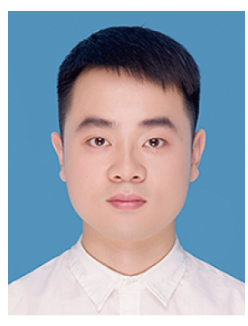

Jie Xu received his BSc degree from the School of Materials Science and Engineering at Tianjin University of Technology, China, in 2016. His current interests include low-dimensional materials and their electron microscopy and optical imaging.

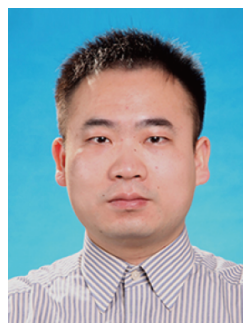

Jun Luo received his BSc (2001) and PhD (2006) degrees from Tsinghua University, China. Then, he worked as a postdoc at Warwick University and a research fellow at Oxford University, UK. In 2011, he joined Tsinghua University as an associate professor. In 2015, he moved to Tianjin University of Technology and is a full professor in the Center for Electron Microscopy. His research interests focus on low-dimensional materials and their electron microscopy and optical imaging.

\section{原子核的 $\mathbf{X}$ 光成像}

许杰, 何佳, 丁轶, 罗俊 ${ }^{*}$

摘要 原子核的光学成像是科学研究和应用研究长期追求的目标, 但迄今为止尚未实现. 本文中, 我们把球差校正的扫描透射电子显 微术(STEM)、X光子的韧致辐射产生以及对这些光子的能量色散 $\mathrm{X}$ 射线光谱(EDS)接收和面扫绘图集成为一种新的光学成像显微方 法, 并通过该方法成功用 $\mathrm{X}$ 光子对不同材料的原子核实现成像. 而 且, 研究表明该方法能用不同的STEM设备实施, 也能区分不同元 素的原子核和分辨小至1皮米量级的原子核成像差异. 因此, 该方 法是一种能对原子核及其在材料科学、化学和物理中的演变进行 成像的通用方法. 